\title{
The Vacuole Binding to Mitochondria by VIG1 Contributes an Equal Inheritance of the Vacuoles in Cyanidioschyzon merolae
}

\author{
Takayuki Fujiwara, Fumi Yagisawa, Mio Ohnuma, Yamato Yoshida, \\ Masaki Yoshida, Keiji Nishida, Osami Misumi, Haruko Kuroiwa and \\ Tsuneyoshi Kuroiwa*
}

Research Information Center for Extremophiles, Rikkyo University, 3-34-1 Nishiikebukuro, Toshimaku, Tokyo 171-8501, Japan

Received March 29, 2010; accepted June 4, 2010

\begin{abstract}
Summary Vacuoles function in endocytosis, storage and digestion of metabolites in eukaryotic cells. They are inherited by the daughter cells. However, the mechanisms of vacuole inheritance are poorly understood because the cells contain multiple vacuoles that behave randomly. Cyanidioschyzon merolae cell has a minimum set of organelles. The vacuoles were equally inherited by the daughter cells by binding to dividing mitochondria. The binding was mediated by VIG1. However, the role of the binding in the vacuoles inheritance was poorly understood. We examined it by inhibiting the binding cytochemically. The vacuoles, which were not bound to mitochondria, were not equally inherited by the daughter cells. As the results, vacuole-less daughter cells were generated. These results suggested that the binding contributed the equal inheritance of vacuoles and ensured the permanence of vacuoles in daughter cells.
\end{abstract}

Key words Lysosome, Mitochondria, Inter-organelle contact, Organelle inheritance, Red alga.

The inheritance of organelles is an essential feature of eukaryotic cell division. In mammals, plants, and yeasts, lysosomes/vacuoles are inherited by the daughter cells (Bergeland et al. 2001, Kutsuna et al. 2003, Han et al. 2003, Weisman 2006). However, the molecular mechanisms were not established because the cells contain multiple vacuoles that behave randomly. Cyanidioschyzon merolae cells offer many advantages for studies of vacuole inheritance. Each $C$. merolae cell has a minimum set of organelles comprising 1 plastid, 1 mitochondrion and a few vacuoles. The organelles are divided in order of a plastid, mitochondrion and a cell nucleus during the cell division (Kuroiwa 1998). The vacuoles are inherited by daughter cells as binding with dividing mitochondria (Misumi et al. 2005, Yagisawa et al. 2007, Fujiwara et al. 2010). These organelle divisions can be highly synchronized by a light/dark cycle (Suzuki et al. 1994).

The transport and binding of vacuoles are not dependent on actin filaments and microtubules (Takahashi et al. 1995, Matsuzaki et al. 2004, Yagisawa et al. 2007). Our previous study reported that the coiled-coil protein VIG1 is essential for the transport and binding of vacuoles during the inheritance in C. merolae (Fujiwara et al. 2010). VIG1 appears on the surface of free vacuoles in the cytosol in the $\mathrm{S}$ phase, and then tethers the vacuoles to the mitochondria during the $\mathrm{G} 2$ and $\mathrm{M}$ phases. The vacuoles are released from the mitochondrion in the daughter cells following VIG1 digestion in early G1 phase. By suppression of VIG1 by antisense RNA, migration of vacuoles was disturbed (Fujiwara et al. 2010). However, a relationship between quantity and function of VIG1 during the processes of vacuole inheritance was not examined. Furthermore, it is thought that the interaction between vacuoles and mitochondria played some kind of important role during the

\footnotetext{
*Corresponding author, e-mail: tsune@rikkyo.ne.jp
} 
inheritance of vacuoles, but the role was poorly understood.

Cell cycle period-specific treatment with a translation inhibitor can suppress expression of VIG1 to various levels, since organelle divisions can be highly synchronized in $C$. merolae. In this study, we examine the function of VIG1 and the inheritance of vacuoles that are not migrated to mitochondria by the treatment of a translation inhibitor.

\section{Materials and methods}

\section{Synchronous culture and cycloheximide treatment}

Cyanidioschyzon merolae 10D-14 were synchronized according to the method of Suzuki et al. (1994). Cells were cultured in 2x Allen's medium at pH 2.3 (Allen 1959). Flasks were shaken under continuous light $\left(40 \mathrm{~W} / \mathrm{m}^{-2}\right)$ at $42^{\circ} \mathrm{C}$. The cells were sub-cultured to $10^{7} \mathrm{cells} / \mathrm{ml}$, and then synchronized by subjecting them to a $12 \mathrm{~h}$ light $/ 12 \mathrm{~h}$ dark cycle at $42^{\circ} \mathrm{C}$ while the medium was aerated. For cycloheximide $(\operatorname{chx})$ treatment, $0.5 \mathrm{M}$ chx dissolved in ethanol was added to synchronized culture at $14 \mathrm{~h}$ at a dilution of $1: 500$. For the control experiment, ethanol was added in same condition. The cells were cultured in medium containing chx and/or ethanol until the cells were harvested for the immunoblotting and immunofluorescent microscopy analyses.

\section{Immunoblotting}

The antisera or antibodies against Dnm1, Dnm2 and VIG1, were previously prepared (Nishida et al. 2003, Miyagishima et al. 2003, Fujiwara et al. 2010). Immunoblotting was performed by conventional methods. C. merolae cells were harvested at each time from initiation of the synchronized culture and lysed in conventional SDS containing sample buffer. Thirty micrograms of proteins of each sample were separated by SDS-PAGE, and then were immunoblotted. The primary antisera or antibodies against Dnm1, Dnm2 and VIG1 were each used at a dilution of $1: 1000$. The secondary antibodies, alkaline phosphatase-conjugated goat anti-guinea pig or antirabbit IgG (Kirkegaad and Perry Laboratories) were each used at a dilution of $1: 3000$.

\section{Fluorescence microscopy}

Cells were harvested during the mitotic phase from synchronized cultures. After fixation and blocking of the cells (Nishida et al. 2004), VIG1 was stained with anti-VIG1 primary antibodies at a dilution of $1: 200$, and secondary goat anti-guinea pig IgG conjugated with Alexa 488 at a dilution of 1:100 (Fujiwara et al. 2010). Vacuoles were stained with anti-V-ATPase primary antibodies at a dilution of $1: 100$ and secondary goat anti-rabbit IgG conjugated with Alexa 555 at a dilution of $1: 100$ (both from Molecular Probes) (Yagisawa et al. 2009). Mitochondria were stained with anti-POR primary antisera at a dilution of $1: 200$, and secondary goat anti-rat IgG conjugated with Alexa 488 at a dilution of 1:300 (Fujiwara et al. 2010). Cells were observed using a fluorescence microscope (Olympus BX51) with a combination of narrow bandpass filter sets: BP470-490 BA510-550 (U-MNIBA2, Olympus) for Alexa-488, BP541-551 BA565-595 (XF37, Omega) for Alexa-555, and using a $\mathrm{Hg}$ arc lamp as a source of excitation light. Images were collected using a 3 charge-coupled device (3CCD) camera system, C7780-10 (Hamamatsu Photonics; Tokyo, Japan) and processed using the M8458-03 RCA-3CCD Photoshop plug-in software. The change of color channels and adjustment of fluorescence and merging of images were performed by Photoshop CS2 (Adobe Systems Inc., California, USA).

\section{Results and discussion}

The cell cycle of $C$. merolae was synchronized by $12 \mathrm{~h}$ light $/ 12 \mathrm{~h}$ dark cycles. Immunofluorescent microscopy showed that behaviors of vacuoles and mitochondria during cell cycle (Fig. 
A Vacuoles/Mitochondria

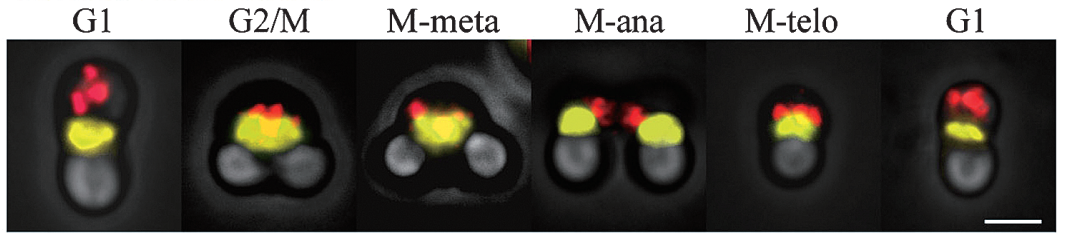

\section{B}
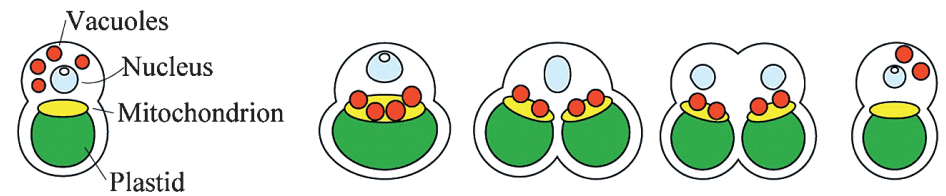

$$
\vdash \mathrm{Gl} \dashv
$$

$$
\vdash \mathrm{G} 2 \longrightarrow
$$

$\mathrm{M}$

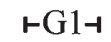

C

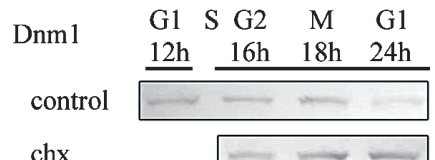

$\operatorname{chx}$

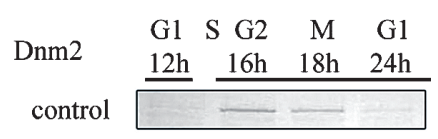

$\operatorname{chx}$

G1 S G2 M G1

VIG1

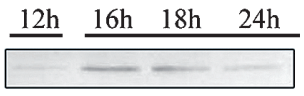

control

$\operatorname{chx}$

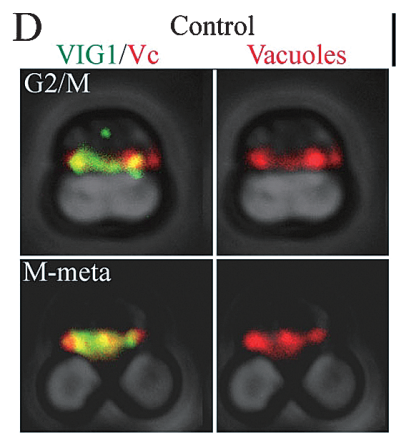

Chx-affected

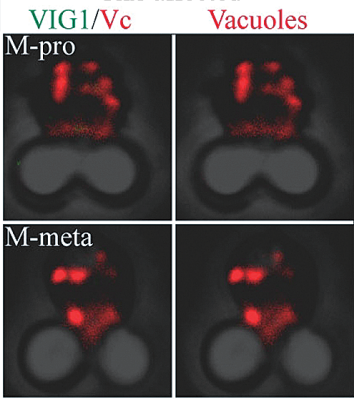

E (\%)

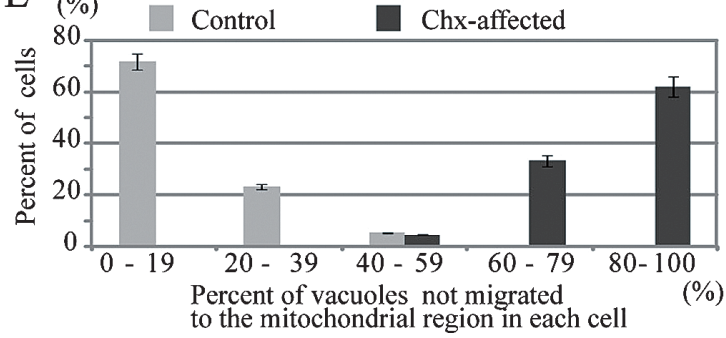

Control

Chx-affected

Fig. 1. The binding between vacuoles and mitochondria was inhibited by cycloheximide treatment targeting VIG1. (A) Phase-contrast and immunofluorescent images showing the inheritance of vacuoles. Vacuoles and mitochondria were shown in red and yellow, respectively. Ethanol was added as a control to chx experiment. (B) Schematic model showing the inheritance of vacuoles. C. merolae cell has 1 cell nucleus, 1 mitochondrion, 1 plastid and a few vacuoles. Vacuoles were located in the nucleus side of cytosol in the G1 phase, and then were transported to the mitochondrial surface by the G2 phase and continued to bind there during the M phase. Vacuoles were transported with divided mitochondria to daughter cells, and then vacuoles were released from the mitochondria in the early G1 of daughter cells. (C) Immunoblotting of Dnm1, Dnm2 and VIG1 during cell cycle in chx-treated cells. The phases of cell cycle and time from onset of synchronous culture were shown on the immunoblot. (D) Phase-contrast and immunofluorescence images showing expression of VIG1 and localization of vacuoles in chx experiment. VIG1 and vacuoles were shown in green and red, respectively. VIG1/Vc, immunofluorescence images of VIG1 overlaid with those of vacuoles. (E) Percentage of the migration of vacuoles in chx-affected cells. The $x$ axis shows categories based on the percentage of vacuoles not migrated to the mitochondria region in each mitotic cell. The $y$ axis shows percent of cells falling in each category. In chx-affected cells, more than forty percent of vacuoles were not migrated to the mitochondrial region. Control: $n=30$; chx-affected: $n=20$. Bars indicate SD. M-meta, M-ana and M-telo shows metaphase, prophase and telophase in the M phase, respectively. Scale bars in $\mathrm{A}$ and $\mathrm{D}=2 \mu \mathrm{m}$. 
A VIG1/Vacuoles

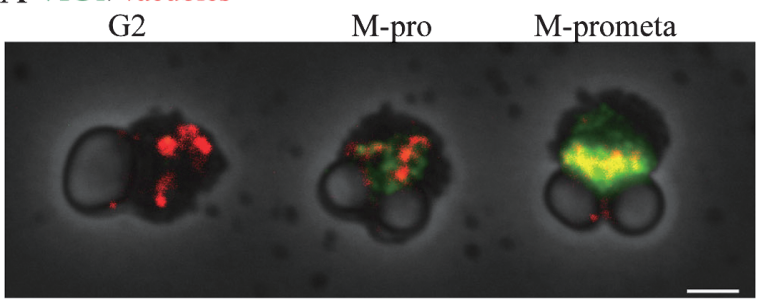

B Vacuoles/Mitochondria
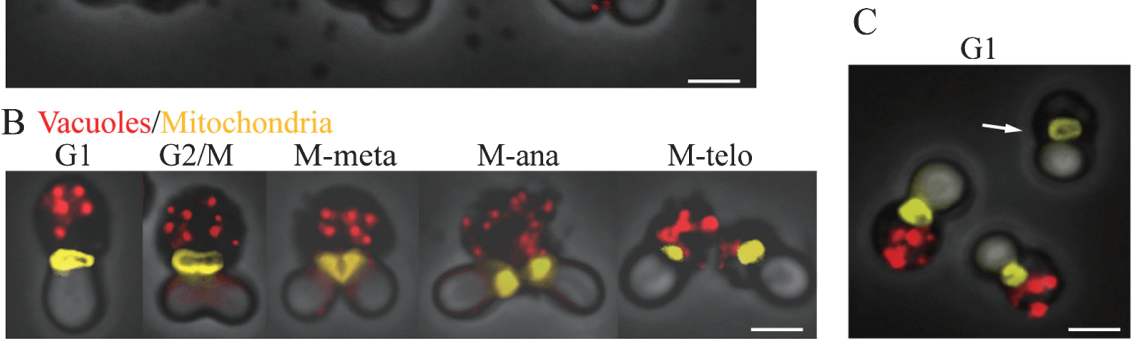

Fig. 2. Phase-contrast and immunofluorescent images showing the inheritance of vacuoles in chx-affected cells. (A) The 3 cells that varied in quantity of VIG1 in a same field. The cells were harvested at $18 \mathrm{~h}$ from onset of synchronous culture. VIG1 and vacuoles were shown in green and red, respectively. The percentage of vacuoles on the mitochondrial region related to quantity of VIG1. (B) The inheritance of vacuoles not migrated to the mitochondria. The cells were harvested at $16 \mathrm{~h}$, $18 \mathrm{~h}$ and $24 \mathrm{~h}$. Vacuoles and mitochondria were shown in red and yellow, respectively, in Fig. 2B and 2C. In the telophase, the vacuoles were unequally inherited. (C) As a result, vacuole-less daughter cells were generated (arrow). M-pro, M-meta, M-ana and M-telo shows metaphase, prophase and telophase in the M phase, respectively. Scale bars $=2 \mu \mathrm{m}$.

1A). They were summarized in a schematic model (Fig. 1B). The vacuoles were distributed in the cytosol during the G1 phase and became bound to the dividing mitochondrion by the G2 phase. During the $\mathrm{M}$ phase, they were inherited by the daughter cells by binding to the dividing mitochondria. In the early G1 phase, the vacuoles were released from the mitochondrion and returned to the cytosol in the daughter cells (Fig. 1A and B).

In $C$. merolae, we already established an antisense-based suppression system. However, the inheritance of vacuoles that were not migrated to mitochondria could not be analyzed, because the cell cycle was not synchronized under the system. We tried, therefore, to inhibit the migration of vacuoles by period-specific inhibition of translation in the synchronous culture and examined the inheritance of vacuole including new daughter-cells after cell division.

To suppress VIG1, cycloheximide (chx), an inhibitor of translation, was added to synchronized cultures at 12,14 or $16 \mathrm{~h}$ at final concentrations of $100 \mu \mathrm{M}$ or $1 \mathrm{mM}$. The time point of $14 \mathrm{~h}$ and concentration of $1 \mathrm{mM}$ were selected as the most efficient combination. To show selectivity for suppression of VIG1 in chx experiment, Dnm1 (dynamin for mitochondrial division constantly expresses during cell cycle) and Dnm2 (dynamin for plastid division mainly express during G2 and M phases) was used as controls. Immunoblotting showed that Dnm1 constantly expressed during cell cycle, Dnm2 and VIG1 mainly expressed in G2 and M phases in control experiment. In chx experiment, Dnm1 was not suppressed and Dnm2 was not completely suppressed, while VIG1 was almost suppressed (Fig. 1C). The cells harvested at $18 \mathrm{~h}$ were fixed and then immunostained. Immunofluorescence microscopy with antibodies against VIG1 and V-ATPase (Vacuole $\mathrm{H}^{+}$. ATPase) showed that the localization of VIG1 and the behavior of vacuoles in chx experiment (Fig. 1D). In control cells, VIG1 was localized at the vacuoles migrated to the mitochondrial region while expression of VIG1 and the migration of vacuoles were suppressed in chx-affected cells (Fig. 1D). Chx did not inhibit the plastid division but the vacuole migration because Dnm2 was not suppressed by chx-treatment (Fig. 1C and D). Statistical data showed that more than half of the vacuoles were not migrated to the mitochondrial region in chx-affected cells (Fig. 1E). The 
migration of vacuoles was successfully inhibited by suppression of VIG1 in chx-treatment (Fig. 1D and $\mathrm{E})$.

To examine period of VIG1 synthesis and relationship between quantity of VIG1 and the behavior of vacuoles, the 3 cells that varied in quantity of VIG1 were shown in a same field (Fig. 2A). Difference in VIG1 quantity was probably caused by the stage of the cell cycle in each cell when chx had been added, because cell cycle stages of individual cells slightly varied within control culture. Vacuoles had been migrated to the mitochondrial surface by the G2 phase in a control (Fig. $1 \mathrm{~A}$ and B), but they did not migrate in chx-treated G2 cells that lacked VIG1 (Fig. 2A G2). In the prophase cell that VIG1 was weakly expressed, the some vacuoles failed to migrate to the mitochondrial region (Fig. 2A M-pro). In the prometaphase cell that VIG1 was substantially expressed, the vacuoles were completely migrated (Fig. 2A M-prometaphase). The G2 cell lacking VIG1 in chx experiment suggested that VIG1 had been synthesized $4 \mathrm{~h}$ before G2 phase. Therefore, the period of VIG1 synthesis was predicted to be S phase according to the examination of temporal relationship among organelle division cycle in the previous study (Imoto et al. 2010). Moreover, the percentage of vacuole on the mitochondrial region related to quantity of VIG1 between the prophase and prometaphase cells, suggesting that VIG1 was also involved in binding between vacuoles and mitochondria.

Furthermore, we examined the inheritance of vacuoles that failed to migrate such as the G2 cell. Immunofluorescent microscopy showed that the migration of vacuoles failed through the cell cycle without inhibiting mitochondrial and plastid division in chx-affected cells (Fig. 2B). In the telophase, the vacuoles, remaining in the cytosol, were not equally inherited by the daughter cells (Fig. 2B M-telo). As a result, vacuole-less daughter cells were generated (Fig. 2C). These results suggested that the binding between vacuoles and mitochondria by VIG1 contributed the equal inheritance of vacuoles, and ensured the permanence of vacuoles in daughter cells.

\section{Acknowledgments}

This work was supported by grants from the Japan Society for the Promotion of Science Fellowships (no. 5061 to T.F.) and for Scientific Research on Priority Areas (no. 19207004 to T.K.); and the Frontier Project "Adaptation and Evolution of Extremophiles" from the Ministry of Education, Culture, Sports, Science and Technology of Japan, and from the program for the Promotion of Basic Research Activities for Innovative Biosciences (PROBRAIN to T.K.).

\section{References}

Allen, M. B. 1959. Studies with Cyanidium caldarium, an anomalously pigmented chlorophyte. Arch Microbiol. 32: $270-277$.

Bergeland, T., Widerberg, J., Bakke, O. and Nordeng, TW. 2001. Mitotic partitioning of endosomes and lysosomes. Curr Biol. 11: 644-651.

Fujiwara, T., Kuroiwa, H., Yagisawa, F., Ohnuma, M., Yoshida, Y., Yoshida, M., Nishida, K., Misumia, O., Watanabe, S., Tanaka, K. and Kuroiwa, T. 2010. The coiled-coil protein VIG1 is essential for tethering vacuoles to mitochondria in vacuole inheritance of Cyanidioschyzon merolae. Plant Cell 22: 772-781.

Han, B. K., Aramayo, R. and Polymenis, M. 2003. The G1 cyclin Cln3p controls vacuolar biogenesis in Saccharomyces cerevisiae. Genetics 165: 467-476.

Imoto, Y., Fujiwara, T., Yoshida, Y., Kuroiwa, H., Maruyama, S. and Kuroiwa, T. 2010. Division of cell nuclei, mitochondria, plastids, and microbodies mediated by mitotic spindle poles in the primitive red alga Cyanidioschyzon merolae. Protoplasma 241: 63-74.

Kuroiwa, T. 1998. The primitive red algae: Cyanidium Caldarium and Cyanidioschyzon merolae as model systems for investigation the dividing apparatus of mitochondria and plastids. BioEssays 20: 344-354.

Kutsuna, N., Kumagai, F., Sato, M. H. and Hasezawa, S. 2003. Three-dimensional reconstruction of tubular structure of vacuolar membrane throughout mitosis in living Tobacco cells. Plant Cell Physiol. 44: 1045-1054. 
Matsuzaki, M., Misumi, O., Shin-I, T., Maruyama, S., Takahara, M., Miyagishima, S. Y., Mori, T., Nishida, K., Yagisawa, F., Nishida, K., Yoshida, Y., Nishimura, Y., Nakao, S., Kobayashi, T., Momoyama, Y., Higashiyama, T., Minoda, A., Sano, M., Nomoto, H., Oishi, K., Hayashi, H., Ohta, F., Nishizaka, S., Haga, S., Miura, S., Morishita, T., Kabeya, Y., Terasawa, K., Suzuki, Y., Ishii, Y., Asakawa, S., Takano, H., Ohta, N., Kuroiwa, H., Tanaka, K., Shimizu, N., Sugano, S., Sato, N., Nozaki, H., Ogasawara, N., Kohara, Y. and Kuroiwa, T. 2004. Genome sequence of the ultrasmall unicellular red alga Cyanidioschyzon merolae 10D. Nature 428: 653-665.

Misumi, O., Matsuzaki, M., Nozaki, H., Miyagishima, S. Y., Mori, T., Nishida, K., Yagisawa, F., Yoshida, Y., Kuroiwa, H. and Kuroiwa, T. 2005. Cyanidioschyzon merolae genome. A tool for facilitating comparable studies on organelle biogenesis in photosynthetic eukaryotes. Plant Physiol. 137: 567-585.

Miyagishima, S. Y., Nishida, K., Mori, T., Matsuzaki, M., Higashiyama, T., Kuroiwa, H. and Kuroiwa, T. 2003. A plantspecific dynamin-related protein forms a ring at the chloroplast division site. Plant Cell 15: 655-665.

Nishida, K., Takahara, M., Miyagishima, S. Y., Kuroiwa, H., Matsuzaki M. and Kuroiwa T. 2003. Dynamic recruitment of dynamin for final mitochondrial severance in a primitive red alga. Proc. Natl. Acad. Sci. USA 100: 2146-2151.

- Misumi, O., Yagisawa, F., Kuroiwa, H., Nagata, T. and Kuroiwa, T. 2004. Triple immunofluorescent labeling of FtsZ, dynamin, and EF-Tu reveals a loose association between the inner and outer membrane mitochondrial division machinery in the red alga Cyanidioschyzon merolae. J. Histochem. Cytochem. 52: 843-849.

Suzuki, K., Ehara, T., Osafune, T., Kuroiwa, H., Kawano, S. and Kuroiwa, T. 1994. Behavior of mitochondria, chloroplasts and their nuclei during the mitotic cycle in the ultramicroalga Cyanidioschyzon merolae. Eur. J. Cell Biol. 63 280-288.

Takahashi, H., Takano, H., Yokoyama, A., Hara, Y., Kawano, S., Toh-e, A. and Kuroiwa, T. 1995. Isolation, characterization and chromosomal mapping of an actin gene from the primitive red alga Cyanidioschyzon merolae. Curr. Genet. 28: 484-490.

Weisman, L. S. 2006. Organelles on the move: Insights from yeast vacuole inheritance. Nat. Rev. Mol. Cell. Biol. 7: $243-252$.

Yagisawa, F., Nishida, K., Kuroiwa, H., Nagata, T. and Kuroiwa, T. 2007. Identification and mitotic partitioning strategies of vacuoles in the unicellular red alga Cyanidioschyzon merolae. Planta 226: 1017-1029.

Yagisawa, F., Nishida, K., Yoshida, M., Ohmura, M., Shimada, T., Fujiwara, T., Yoshida, Y., Misumi, O., Kuroiwa, H. and Kuroiwa, T. 2009. Identification of novel proteins in isolated polyphosphate vacuoles in the primitive red alga Cyanidioschyzon mololae. Plant J. 60: 882-893. 